\title{
Necessity for a paradigm shift in the treatment of pediatric obesity
}

\author{
J. C. $\operatorname{Han}^{1} \cdot$ R. L. Atkinson $\mathbb{1}^{2} \cdot$ I. A. Macdonald ${ }^{3} \cdot$ A. Pietrobelli ${ }^{4,5}$
}

Received: 17 September 2018 / Accepted: 18 September 2018 / Published online: 9 October 2018

(c) Springer Nature Limited 2018

Since 2004, the International Journal of Obesity (IJO) has been showcasing research on pediatric obesity in its quarterly Pediatric Highlights section. The importance of preventing and treating childhood obesity has garnered considerable global attention from not only clinicians and researchers, but also the media, lawmakers, and the general public. Despite this outpouring of interest and investment and the numerous studies that have been conducted, advances in this field unfortunately have been incremental, raising important questions about our current research strategies.

In this issue of $I J O$, Ells et al. [1] provide an updated review of the extant evidence for the treatment of children and adolescents with overweight and obesity, examining findings from randomised controlled trials, comprising one surgical, 21 pharmacological, and 141 behavioural studies. The authors conclude that "multi-component behaviour changing interventions may be beneficial in achieving small reductions in body weight status in children of all ages" while the data for bariatric surgery and weight loss medications are "too limited to make inferences about use and safety." These findings are disheartening in that they are nearly identical to those of a similar Cochrane review from a decade ago [2]. Nor has there been significant advancement since the European perspective on childhood obesity was published in 2004, which underscored the importance

$\triangle$ A. Pietrobelli

angelo.pietrobelli@univr.it

1 Departments of Pediatrics and Physiology, University of Tennessee Health Science Center and Le Bonheur Children's Foundation Research Institute, Memphis, TN, USA

2 Virginia Commonwealth University, Richmond, VA, USA

3 School of Life Sciences, Queen's Medical Centre, University of Nottingham, Nottingham NG7 2UH, UK

4 Verona Pediatric Clinic, Verona University Medical School, Verona, Italy

5 Pennington Biomedical Research Center, Baton Rouge, LA, USA of elucidating "nutritional factors, energy intake and composition of the diet, nutrition and hormonal status, food preferences and behaviour, and the influence of nonnutritional factors" for addressing this epidemic [3].

We have also included in this issue of $I J O$, a meeting report by Kelly et al. [4] summarising the proceedings of the United States National Institutes of Health (NIH)-sponsored workshop convened to address this dearth of progress in identifying effective and safe treatment options for severe childhood obesity. The authors reach similar conclusions about the lacklustre performance of lifestyle interventions as stand-alone therapy, particularly in adolescents with extreme obesity, and they call for "precision medicine approaches" and enhanced "methodical rigor in pediatric obesity research."

Meaningful progress will require a paradigm shift. Most clinical trials are currently designed to exclude patients with known aetiologies of obesity in order to identify treatments that are broadly generalizable. However, these one-size-fitsall approaches are largely ineffective. In contrast, substantial weight loss has been observed in patients with specific genetic defects, such as leptin replacement for patients with leptin gene mutations [5] and melanocortin-4 receptor agonist therapy for patients with leptin receptor [6] and proopiomelanocortin [7] gene mutations. While individuals affected with these conditions are exceedingly rare, common variants in these and other genes that regulate appetite, energy expenditure, and adipocyte differentiation could play important roles in determining clinical response to interventions. With genetic testing becoming more economical and widely available, pharmacogenomics, which has already entered standard practice in oncology, should be applied to other specialties, including obesity.

Beyond the necessity for additional genotype-specific obesity therapies to be developed, more basic science studies are needed in embryologic and juvenile model organisms to identify novel developmental determinants of energy homeostasis that can also serve as therapeutic targets. Further mechanistic studies are also required to elucidate the physiologic mechanisms (e.g., potential roles of 
incretins, afferent neuronal pathways, meal size and timing, microbiome changes, bile acid profile, epigenetic modifications, etc.) by which bariatric surgery exerts its benefits so that non-surgical therapies that mimic these effects can be pursued. Most importantly, additional funding to support obesity research is essential. In 2017, NIH-sponsored research funding for obesity was only one-sixth that of cancer despite the greater than three-fold higher prevalence of obesity compared to cancer [8]. Considering the contribution of obesity to increased cancer-related mortality (with 19\% higher risk of death due to cancer for every $5 \mathrm{~kg} / \mathrm{m}^{2}$ increase in BMI [9]), targeting obesity would reduce the disease burden of cancer as well as many other conditions exacerbated by body fatness. Furthermore, Jensen et al. [10] report in this issue of IJO that "childhood overweight that persists into early adulthood is associated with an increased risk of colon cancer, whereas overweight that disappears before early adulthood or developed after childhood is not."

While lifestyle behaviours will always remain important at the core of health promotion, if we simply continue to revisit behaviour modification interventions in various myriad permutations, progress in combatting childhood obesity will be hindered. Generalized approaches to pharmacotherapy will also continue to have limited impact. Since the dawn of the leptin era [11], publications on energy homeostasis regulation have exponentially expanded, but practical solutions for the obesity epidemic have yet to come to fruition. While leptin proved to be highly effective for treating leptin deficiency, it has not been a panacea for the remaining forms of obesity, and no single drug will likely ever be the answer. Researchers, clinicians, and policy makers must redirect efforts to address the developmental origins of obesity and seek novel, individualized treatments rather than revisiting standard protocols and approaches. After all, we can hardly expect to discover new paths if we only trod in the places we have already journeyed.

\section{Compliance with ethical standards}

Conflict of interest JCH is a clinical trial investigator for a Rhythm Pharmaceuticals sponsored study of a medication to treat the obesity observed in rare genetic disorders. RLA is a consultant to Novo
Nordisk. IAM is a member of Scientific Advisory Boards for Mars Inc., Nestle, IKEA, Zaluvida, and a member of the Scientific Advisory Committee on Nutrition for the UK Government's Public Health England. The remaining author declares that he has no conflict of interest.

\section{References}

1. Ells LJ, Rees K, Brown T, Mead E, Al-Khudairy L, Azevedo L et al. Interventions for treating children and adolescents with overweight and obesity: an overview of Cochrane reviews. Int $\mathbf{J}$ Obes. 2018.

2. Oude Luttikhuis H, Baur L, Jansen H, Shrewsbury VA, O'Malley C, Stolk RP et al. Interventions for treating obesity in children. Cochrane Database Syst Rev. 2009; CD001872.

3. Flodmark CE, Lissau I, Moreno LA, Pietrobelli A, Widhalm K. New insights into the field of children and adolescents' obesity: the European perspective. Int $\mathrm{J}$ Obes Relat Metab Disord. 2004;28:1189-96.

4. Kelly AS, Marcus MD, Yanovski JA, Yanovski SZ, Osganian SK. Working toward precision medicine approaches to treat severe obesity in adolescents: report of an NIH workshop. Int J Obes. 2018.

5. Farooqi IS, Jebb SA, Langmack G, Lawrence E, Cheetham $\mathrm{CH}$, Prentice AM, et al. Effects of recombinant leptin therapy in a child with congenital leptin deficiency. N Engl J Med. 1999;341: 879-84.

6. Clement K, Biebermann H, Farooqi IS, Van der Ploeg L, Wolters $\mathrm{B}$, Poitou $\mathrm{C}$, et al. MC4R agonism promotes durable weight loss in patients with leptin receptor deficiency. Nat Med. 2018;24: $551-5$.

7. Kuhnen P, Clement K, Wiegand S, Blankenstein O, Gottesdiener $\mathrm{K}$, Martini LL, et al. Proopiomelanocortin deficiency treated with a melanocortin-4 receptor agonist. N Engl J Med. 2016;375: $240-6$.

8. National Institutes of Health Research Portfolio Online Reporting Tools. Estimates of Funding for Various Research, Condition, and Disease Categories; https://report.nih.gov/categorical_spending. aspx (accessed 11 September 2018).

9. Global BMIMC, Di Angelantonio E, Bhupathiraju ShN, Wormser D, Gao P, Kaptoge S, et al. Body-mass index and allcause mortality: individual-participant-data meta-analysis of 239 prospective studies in four continents. Lancet. 2016;388: 776-86.

10. Jensen BW, Bjerregaard LG, Angquist L, Gogenur I, Renehan $\mathrm{AG}$, Osler $\mathrm{M}$ et al. Change in weight status from childhood to early adulthood and late adulthood risk of colon cancer in men: a population-based cohort study. Int J Obes. Accessed on 22 May 2018. https://doi.org/10.1038/s41366-018-0109-y.

11. Zhang Y, Proenca R, Maffei M, Barone M, Leopold L, Friedman JM. Positional cloning of the mouse obese gene and its human homologue. Nature. 1994;372:425-32. 